\section{A New Culture Medium for in Vitro Rhizogenesis of Grapevine (Vitis spp.) Genotypes}

\author{
K.A. Roubelakis-Angelakis' and S.B. Zivanovitc ${ }^{2}$ \\ University of Crete, P. O. Box 1470, 71110 Heraklion, Greece \\ Additional index words. tissue culture, rooting
}

\begin{abstract}
A modified culture medium is presented that promotes in vitro rooting of grapevine rootstock and Vitis vinifera $L$. cultivars even in the absence of plant growth regulators. Study of 15 Vitis genotypes indicated a strong genotype-dependent response to culture medium and growth regulators with respect to formation of roots in onenode shoot segments.
\end{abstract}

In vitro micropropagation of woody plant species is an increasingly applied practice. The feasibility depends on the shoot multiplication rate from subculture shoots and the percentage rooting of either the shoots produced or one-node green shoot segments.

Rapid micropropagation of grapevine can be achieved not only by combining shoot proliferation and rooting of new shoots, but also by sequential rooting of one-node shoot segments from in vitro or greenhouse-grown vines (Alleweldt and Radler, 1962; Roubelakis-Angelakis and Katsirdakis, 1990). The latter results in lower multiplication rates, but it avoids the use of cytokinins, which may result in higher incidence of undesired somaclonal variation (Beauchesne, 1982).

Rhizogenesis in explants of some grapevine genotypes does not always require the presence of auxins (Barlass and Skene, 1980; Grenan, 1977); addition of $1 \mathrm{H}$-indole-3-acetic acid (IAA), 1-napthaleneacetic acid (NAA), or $1 \mathrm{H}$-indole-3-butanoic acid (IBA) at 0.1 to $2 \mu \mathrm{M}$ was sufficient for root primordia induction, but root growth was enhanced when the salt concentration was reduced in the medium (Chee and Pool, 1983; Grenan, 1977; Rajasekaran and Mullins, 1981). Rooting percentages of $V$ vinifera explants were superior with IAA or IBA than with NAA, whereas the opposite occurred with $\mathrm{V} \mathrm{ru}$ pestris explants (Chee and Pool, 1983, 1987).

We present a systematic study of the rhizogenic behavior of several economically important $V$. vinifera cultivars and interspecific hybrids that are used as rootstock worldwide and we provide information on a new modification of a culture medium that significantly promotes, even in the absence of exogenous plant growth regulators, rhizogenesis and shoot elongation in most of the tested genotypes.

Received for publication 21 Dec. 1989. This work was supported by the Greek Ministry of Industry, Energy and Technology and the SFS Programme. The cost of publishing this paper was defrayed in part by the payment of page charges. Under postal regulations, this paper therefore must be hereby marked advertisement solely to indicate this fact. 'Dept. of Biology.

${ }^{2}$ Institute of Molecular Biology and Biotechnol. ogy.
Genotypes. We used $V$. vinifera cultivars Cabernet franc, Loose Perlette, Sultanina, Italia, Cardinal, Rhazaki (a Greek table grape), and Liatiko and Mandilari (Greek wine cultivars). The following rootstock cultivars were also used: 'ARG No. 1' (Aramon $x$ three experiments. Bars represent SE.
Rupestris Ganzin. No. 1), 'Couderc 1613' (Othello (labrusca-riparia-vinifera) $\times$ Solonis ( riparia-rupestris-candicans), 'Richter 99' and 'Richter 110' ( $V$. berlandieri $\times$ $V$. rupestris), 'S04' ( V. berlandieri $\times \mathrm{V}$ riparia), 'Rupestris du Lot' ( $V$. rupestris) and 'Salt Creek' ( V. champini).

Plant material. Plant material was from greenhouse- or in vitro-grown vines. All vines were determined to be free of grapevine fanleaf virus, the leafroll-associated closteroviruses, stem pitting, and corky bark (Theodoropoulos and Roubelakis-Angelakis, 1990). One-node, 1-to 2-cm-long shoot segments were collected from the apical 15 nodes of green shoots of own-rooted vines. They were surface sterilized with $70 \%(\mathrm{v} / \mathrm{v})$ ethanol for 3 rein, 3\% (w/v) Ca-hypochlorite containing $0.1 \%(\mathrm{v} / \mathrm{v})$ Tween-20 for 10 rein, and rinsed with sterile distilled water three times. Also, mother vines were aseptically grown in hormone-free Roubelakis medium (described below) at $25 \pm 1 \mathrm{C}$, under a 16-h photoperiod with $50 \mu \mathrm{mol} \cdot \mathrm{m}^{-2} \cdot \mathrm{s}^{-1}$ total energy provided by cool-white fluorescent
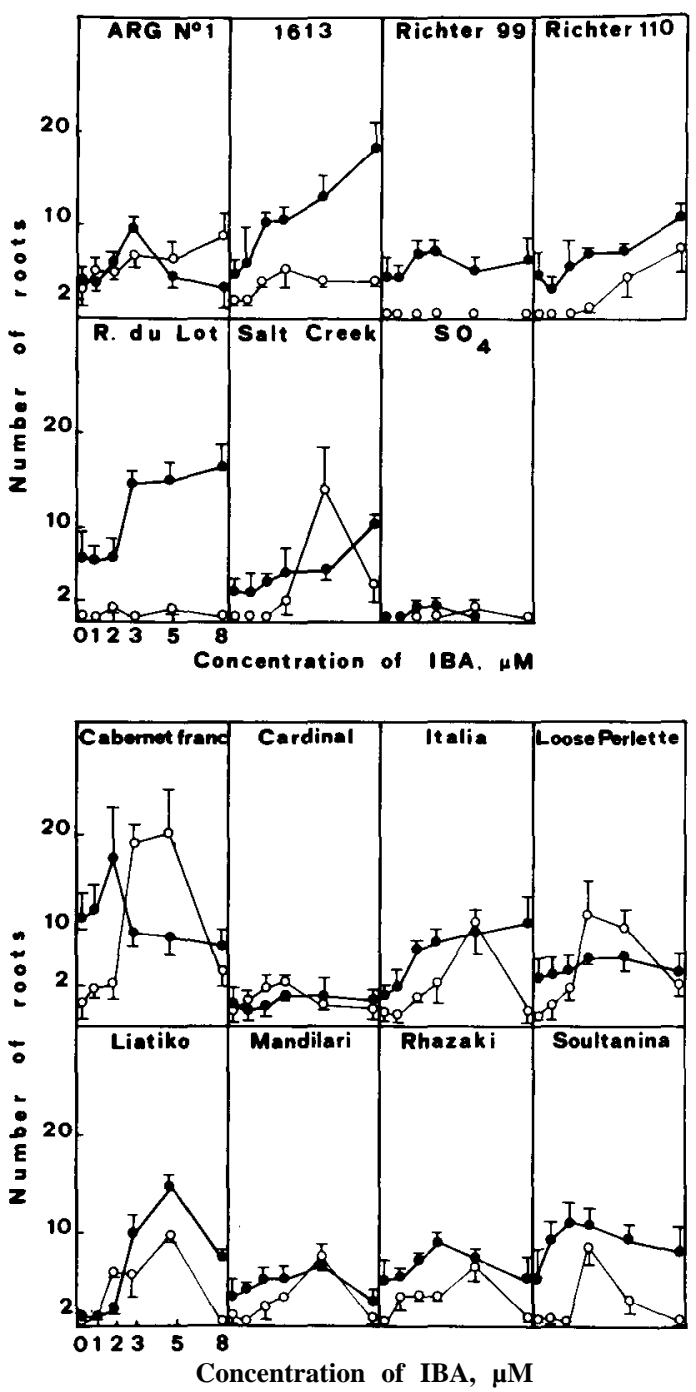

Fig. 1. Number of roots per one-node segment of seven Vitis rootstock (top) and eight Vitis vinifera (bottom) cultivars in response to Murashige and Skoog ( $O$ ), and Roubelakis ( ) culture media at various IBA concentrations. Each point represents the mean of five replicates per treatment from 

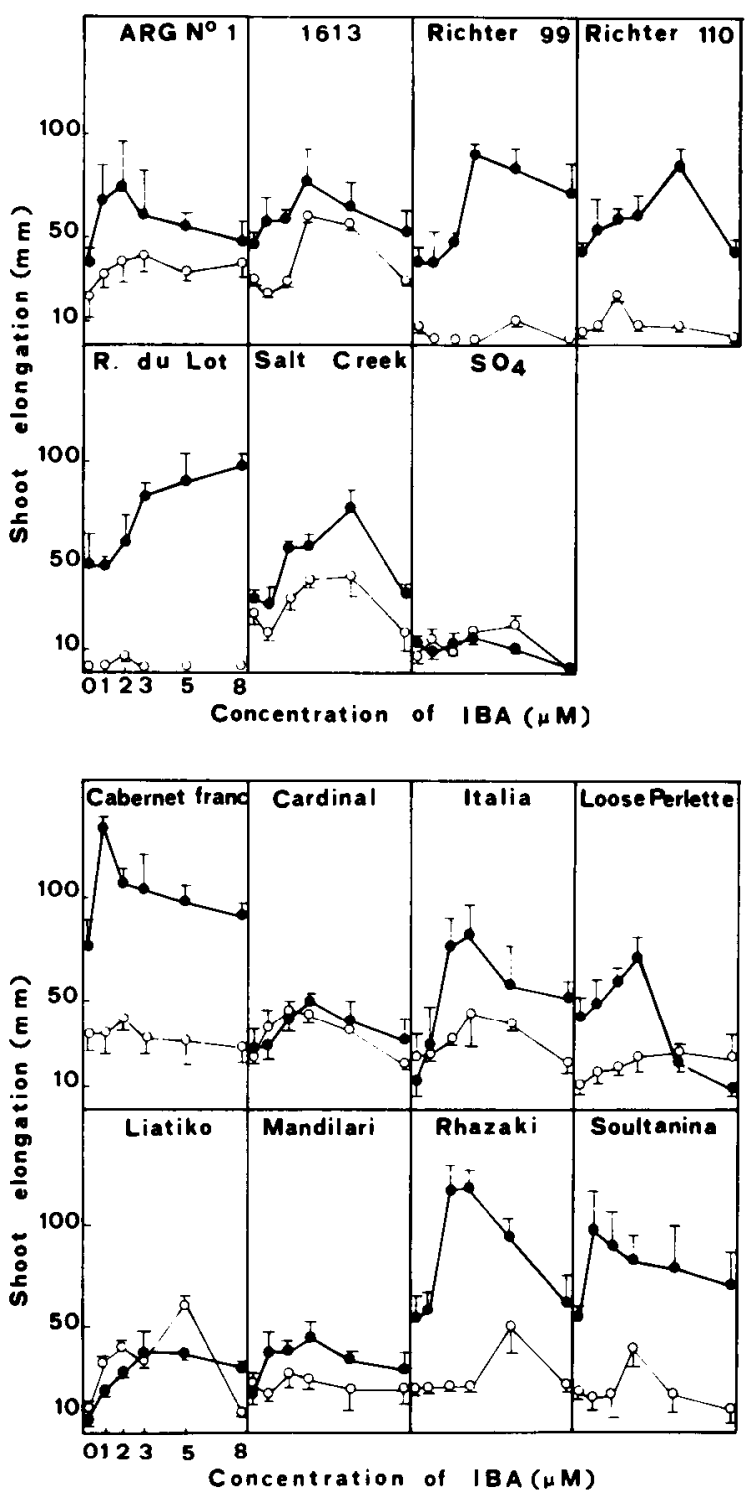

Fig. 2. Shoot elongation of rooted explants of seven Vitis rootstock (top) and eight Vitis vinifera (bottom) cultivars in response to Murashige and Skoog (O), and Roubelakis (•) culture media at various IBA concentrations. Each point represents the mean of five replicates per treatment from three experiments. Bars represent SE.

lamps. One-node shoot segments were handled aseptically. The culture tubes $(20 \times 3$ $\mathrm{cm})$ with $25 \mathrm{ml}$ of culture medium were plugged with cotton and covered with aluminum foil.

Culture media and conditions. The media used were Murashige and Skoog (MS) (Murashige and Skoog, 1962), pH 5.7; and a medium, we have designated Roubelakis, containing (in mg.liter ${ }^{-1}$ ): $\mathrm{NH}_{4} \mathrm{NO}_{3} 500$, $\mathrm{KNO}_{3} 1000, \mathrm{CaCl}_{2} \cdot 2 \mathrm{H}_{2} \mathrm{O} 200, \mathrm{MgSO}_{2} \cdot 7 \mathrm{H}_{2} \mathrm{O}$ $180, \mathrm{KH}_{2} \mathrm{PO}_{4} 100, \mathrm{MnSO}_{4} \cdot 7 \mathrm{H}_{2} \mathrm{O} 5, \mathrm{H}_{3} \mathrm{BO}_{3}$ $1, \mathrm{ZnSO}_{4} \cdot 7 \mathrm{H} 2 \mathrm{O} 1, \mathrm{KI} 0.5, \mathrm{CuSO}_{4} \cdot 5 \mathrm{H}_{2} \mathrm{O} 0.01$, $\mathrm{CoCl} \cdot 6 \mathrm{H}_{2} \mathrm{O}$ 0.01, EDTA 40, biotin 0.1, nicotinic acid 5, pyridoxine 5, thiamine 5 , pantothenic acid 5, myo-inositol 100, sucrose $2 \%(\mathrm{w} / \mathrm{v})$, and agar $0.7 \%(\mathrm{w} / \mathrm{v}), \mathrm{pH}$ 6.4. IBA was added at $1,2,3,5,8 \mu \mathrm{M}$. The effects of the antioxidant citric acid at $1 \mu \mathrm{M}$ and of activated charcoal at $3 \%(\mathrm{w} / \mathrm{v})$ were also tested. Other factors studied were liquid vs. solid medium; light vs. semi- and complete darkness; and one-tenth and onehalf vs. full strength MS medium. The standard incubation conditions described in the previous section were used. The experimental period was 42 days. The number of roots per explant and shoot elongation were recorded. Each treatment consisted of five replicates of three explants each. Each experiment was repeated at least three times. Data were subjected to factorial analysis of variance by using the Statgraphics PC Program (statistical Graphics Corp., version 2.6, serial no. 599458).

Plant acclimation. Plants with shoots 10 to $15 \mathrm{~cm}$ long were planted in plastic cups in peat and kept under high humidity. The plants were watered daily and gradually exposed to open air during 7 to 10 days. Then, they were transplanted and transferred to the greenhouse.

In preliminary experiments on rhizogenesis, IBA gave superior results to IAA, NAA, and 2,4-D and was further used in this work. The number of roots forming on onenode segments in the rootstock (Fig. 1, top) and $V$. vinifera (Fig. 1, bottom) cultivars was determined. Of the 15 genotypes that we studied, seven did not form roots in MS me- dium without IBA, whereas the remaining eight developed poor 'root systems (Fig. 1). In Roubelakis medium without IBA, almost all genotypes developed strong root systems, with the exception of 'Liatiko' and 'S04', which performed poorly in both media in the absence (Liatiko) or in the presence (S04) of IBA.

Among the rootstock (Fig. 1, top), the observed differences between the two tested culture media were more pronounced than for the cultivars (Fig. 1, bottom); S04 had the lowest rooting frequency in both media, whereas 'Richter 99' did not root at all in MS medium at any IBA concentration and 'Rupestris du Lot' exhibited very low rhizogenic response in the same medium. However, 'Richter 99' and 'Rupestris du Lot' rooted very well on Roubelakis medium at all IBA concentrations. Optimum IBA concentrations ranged from 3 to $8 \mu \mathrm{M}$. Most of these genotypes also exhibited statistically superior $(P=0.05)$ rooting frequency on Roubelakis medium in the absence of exogenous auxins.

Of the $V$. vinifera cultivars, Cabernet franc exhibited the highest and Cardinal the lowest rhizogenic response in both tested culture media (Fig. 1, bottom). The optimum IBA concentration for $V$. vinifera cultivars ranged from 3 to $5 \mu \mathrm{M}$. In most genotypes, these concentrations coincided in the two culture media. However, in nearly all cultivars at zero auxin concentration, a higher percentage of explants forming roots was found in Roubelakis medium.

Total shoot elongation in one-node segments varied among genotypes and treatments (Fig. 2). During the 42-day in vitro growing period, shoot elongation ranged from 10 to $150 \mathrm{~mm}$. In most of the tested genotypes, shoot elongation was significantly superior in Roubelakis than in MS medium. Shoot elongation with varying IBA concentrations paralleled the rhizogenic response.

In general, plants were better developed on Roubelakis medium and callus formation at the basal end of the explant was rare compared with that on MS medium. Also, higher concentrations of IBA caused the development of thicker roots and very few secondary roots, whereas absence of IBA or lower concentrations promoted development of roots with normal appearance and numerous secondary roots and root hairs. In MS medium, optimum overall rhizogenic response in all genotypes was found at $5 \mu \mathrm{M}$ IBA and leveled off at higher concentrations, whereas in Roubelakis medium, response to concentrations $>3 \mu \mathrm{M}$ remained about constant.

The overall ranking of the 15 genotypes studied, from the highest to the lowest percentage of root formation, was: 'ARG No. 1', 'Cabernet franc', 'Loose Perlette', 'Richter 110', 'Mandilari', 'Salt Creek', 'Sultanina', 'Italia', 'Rhazaki', 'Cardinal', 'Couderc 1613', 'Liatiko', 'Richter 99', 'SO4' and 'Rupestris du Lot'.

Addition of the antioxidant citric acid at $1 \mu \mathrm{M}$ or of activated charcoal at $3 \%$ in MS medium did not increase the number of onenode segments producing roots; to the con- 


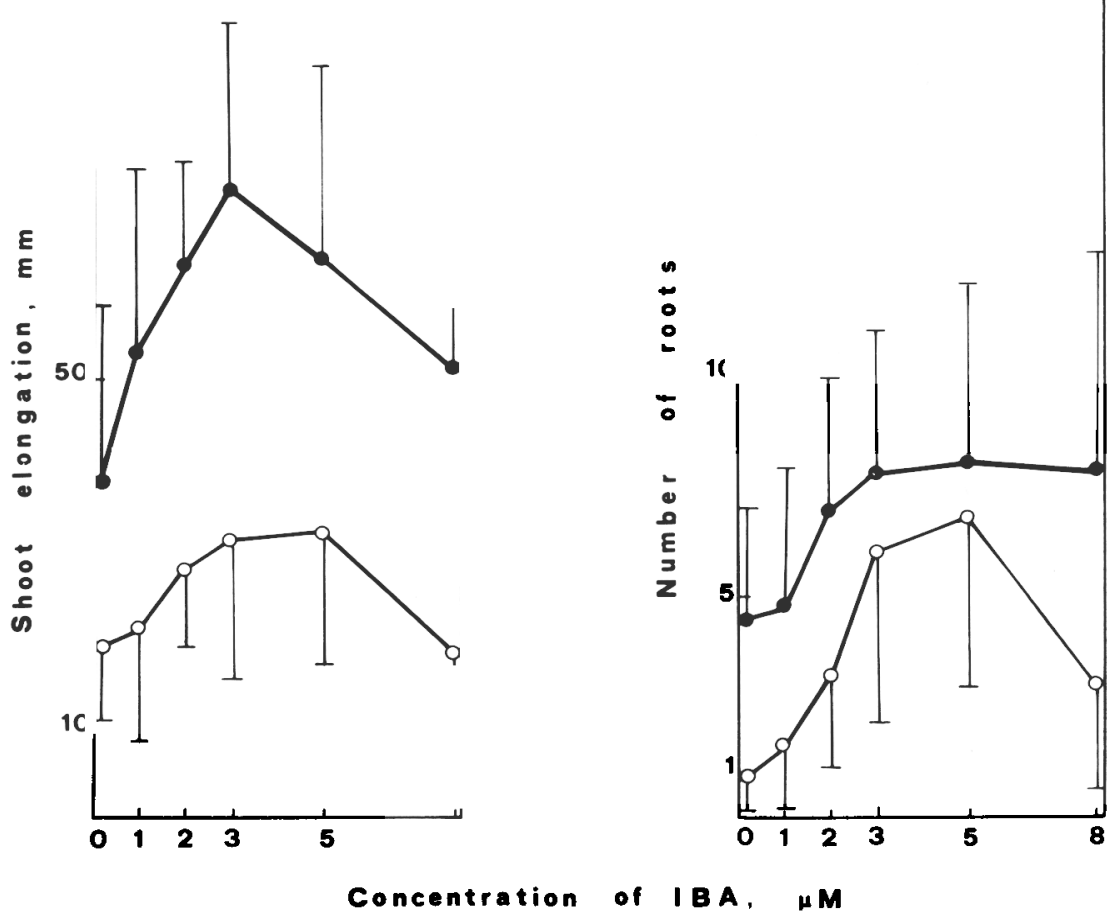

Fig. 3. Mean shoot elongation (left) and number of roots (right) of rooted explants of 15 Vitis spp. genotypes in Murashige and Skoog $(\circ)$, and Roubelakis $(\bullet)$ media at various IBA concentrations. Bars represent SE.

trary, citric acid reduced the number of respending explants (data not shown). Also, use of one-tenth MS medium resulted in an early development of chlorotic symptoms in developing leaves. Further, root initiation and root elongation on solid medium were superior to that on liquid (data not shown).

Roubelakis medium clearly was more effective than MS medium, especially in the absence of exogenous auxin, in enhancing shoot elongation (Fig. 3, left) and root formation (Fig. 3, right).

Previous reports have proposed the use of full- or half-strength MS or Nitsch and Nitsch (1967) medium for rooting of shoots of Vitis genotypes, supplemented with $0.5 \mu \mathrm{M}$ NAA (Barlass and Skene, 1980), or $0.1 \mu \mathrm{M} \mathrm{IBA}$ (Novak and Juvova, 1983), or $5 \mu \mathrm{M}$ 2,4-D and nicotinic acid (Rajasekaran and Mullins, 1981). Addition of only 2,4-D failed to induce rhizogenesis (Novak and Juvova, 1983). In two interspecific hybrids, 'Marechal Foch' and 'Cascade' shoot explants failed to develop roots in MS medium without exogenous auxin (Li and Eaton, 1984).

Compared with MS medium, Roubelakis medium contains significantly less ammonium nitrate (500 vs. $1650 \mathrm{mg} \cdot$ liter $^{-1}$ ), about half the $\mathrm{KNO}_{3}, \mathrm{Ca}$, and $\mathrm{Mg}(1000$ vs. 1900 , 200 vs. 440 , and 180 vs. $370 \mathrm{mg} \cdot$ liter $^{-1}$, respectively); significantly reduced microelement concentrations; lower sucrose $(2 \%$ vs. 3\%); contains biotin and pantothenic acid and has-higher $\mathrm{pH}$ (6.4 vs. 5.5). Roubelakis medium was superior to MS medium at $\mathrm{pH}$ 6.4 , or with sucrose at $2 \mathrm{~g} \cdot \mathrm{liter}^{-1}$ (data not shown). Therefore, it seems that the increased rhizogenic effect of this medium is a combined effect brought about by its modified composition and not simply by the higher $\mathrm{pH}$ or the lower sucrose content. The observation that Roubelakis medium can induce rhizogenesis in one-node green shoot explants from grapevine genotypes without exogenous auxin is of special importance, because absence of plant growth regulators reduces the possibility of genetic instability during in vitro micropropagation (Beauchesne, 1982). Root elongation also has been reported to be more rapid on hormone-free medium (Hicks, 1980; Goussard, 1981).

Mean shoot elongation of the 15 genotypes studied showed parallel patterns in the two media tested. At all tested IBA concentrations, shoot elongation was significantly superior in Roubelakis medium than in fullstrength MS medium (Fig. 3, left). Thus, we recommend this medium for in vitro rooting of one-node green shoot segments of all tested Vitis genotypes. The use of activated charcoal and citric acid was not justified, in agreement with previous work (Novak and Juvova, 1983).

In conclusion, a strong genotype-depen- dent rhizogenic response of grapevine to culture medium and auxins was shown. In addition, a modified culture medium was introduced that can induce formation of roots in the absence of auxins or development of more roots in the presence of 3 to $5 \mu \mathrm{M} \mathrm{IBA}$.

\section{Literature Cited}

Alleweldt, G. and F. Radler. 1962. Interrelationship between photoperiodic behavior of grapes and growth of plant tissue cultures. Plant Physiol. 37:376-379.

Barlass, M. and K.G.M. Skene. 1980. Studies on the fragmented shoot apex of grapevine. II. Factors affecting growth and differentiation. J. Expt. Bet. 31:489-495.

Beauchesne, G. 1982. Appearance of plants not true to type during in vitro plant propagation, p. 268-272. In: E.D. Earle and Y. Demarly (eds.). Variability in plants regenerated from tissue culture. Praeger Publications, New York.

Chee, R. and R.M. Pool. 1983. In vitro vegetative propagation of Vitis: Application of previously defined culture conditions to a selection of genotypes. Vitis 22:363-374.

Chee, R. and R.M. Pool. 1987. Improved inorganic media constituents for in vitro shoot multiplication of Vitis. Scientia Hort. 32:85-95.

Goussard, P.G. 1981. Effects of eytokinins on elongation, proliferation and total mass of shoots derived from shoot apices of grapevine cultured in vitro. Vitis 20:228-234.

Grenan, S. 1977. Rhizogenese de bourgeons apicaux de boutures de vigne cultives in vitro. Connaissance Vigne Vin. 13:125-136.

Hicks, G.S. 1980. Patterns of organ development in plant tissue culture and the problem of organ determination. Bet. Rev. 46:1-23.

Li, J.R. and G.W. Eaton. 1984. Growth and rooting of grape shoot apices in vitro. HortScience 19:64-66.

Murashige, L and F. Skoog. 1962. Revised medium for rapid growth and bioassay with tobacco tissue cultures. Physiol. Plant. 15:473497.

Nitsch, C. and J.P. Nitsch. 1967. The induction of flowering in vitro in stem segments of Plumbage indica $\mathrm{L}$. $\mathrm{L}$ The production of vegetative buda. Planta 72:355-370.

Novak, F.J. and Z. Juvova. 1983. Clonal propagation of grapevine through in vitro axillary bud culture. Scientia Hort. 18:231-240.

Rajasekaran, K. and M.G. Mullins. 1981. Organogenesis in internode explants of grapevines. Vitis 20:218-227.

Roubelakis-Angelakis, K.A. and K.C. Katsirdakis. 1990. In vitro micromultiplication of grapevine: Effect of age, genotype and culture conditions on induction of callus in Vitis spp. leaf segments. p. 89-95. In: R. Rodrigues, R. Tames, and D.J. Durzan (eds.). Plant aging: basic and applied approaches. Kluwer Academic, New York.

Theodoropoulos, P.A. and K.A. Roubelakis-Angelakis. 1990. Progress in leaf protoplasts isolation and culture from virus-free axenic shoot cultures of Vitis vinifera L. Plant Cell Tissue Oman Culture 20:15-23. 